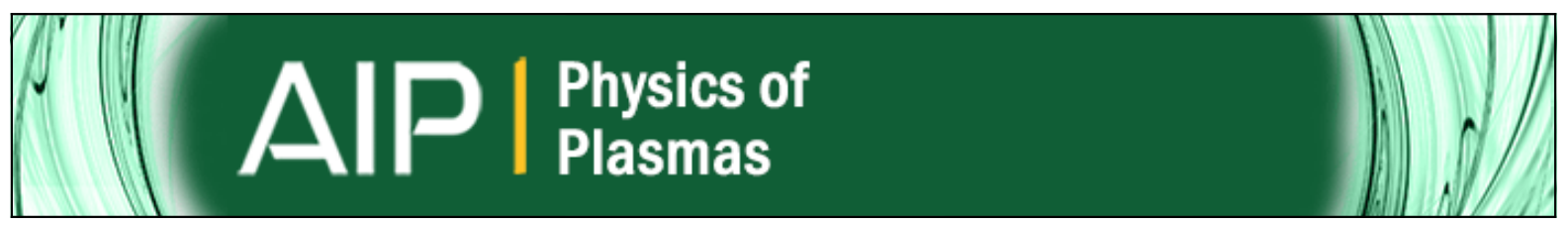

\title{
Mixing and space-charge effects in free-electron lasers
}

E. Peter, A. Endler, F. B. Rizzato, and A. Serbeto

Citation: Physics of Plasmas (1994-present) 20, 123104 (2013); doi: 10.1063/1.4841375

View online: http://dx.doi.org/10.1063/1.4841375

View Table of Contents: http://scitation.aip.org/content/aip/journal/pop/20/12?ver=pdfcov

Published by the AIP Publishing

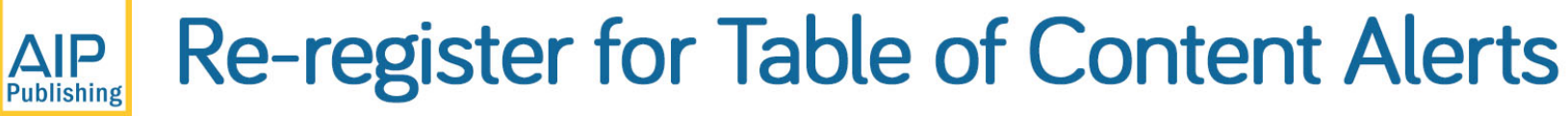

Create a profile.

Sign up today! 


\title{
Mixing and space-charge effects in free-electron lasers
}

\author{
E. Peter, ${ }^{1, a)}$ A. Endler, ${ }^{1, b)}$ F. B. Rizzato, ${ }^{1, c)}$ and A. Serbeto ${ }^{2, d)}$ \\ ${ }^{1}$ Instituto de Física, Universidade Federal do Rio Grande do Sul, Caixa Postal 15051, \\ 91501-970 Porto Alegre, RS, Brazil \\ ${ }^{2}$ Instituto de Física, Universidade Federal Fluminense, Av. Gal. Milton Tavares de Souza s/n, \\ Campus da Praia Vermelha, 24210-346 Niterói, RJ, Brazil
}

(Received 4 October 2013; accepted 22 November 2013; published online 9 December 2013)

\begin{abstract}
The present work revisits the subjects of mixing, saturation, and space-charge effects in free-electron lasers. Use is made of the compressibility factor, which proves to be a helpful tool in the related systems of charged beams confined by static magnetic fields. The compressibility allows to perform analytical estimates of the elapsed time until the onset of mixing, which in turn allows to estimate the saturated amplitude of the radiation field. In addition, the compressibility helps to pinpoint space-charge effects and the corresponding transition from Compton to Raman regimes. (C) 2013 AIP Publishing LLC. [http://dx.doi.org/10.1063/1.4841375]
\end{abstract}

\section{INTRODUCTION}

Free-electron lasers (FELs) are devices designed to efficiently convert the kinetic energy of electron beams into the energy of coherent electromagnetic modes. Energy is drawn from the electron beam, as electrons are trapped in the ponderomotive well formed by the simultaneous action of the electromagnetic mode and wiggler field. ${ }^{1-3}$

FELs are complex systems where electrons not only interact with the growing electromagnetic modes, but also among themselves. The regime, where the self-interaction of the electronic population is negligible, is called Compton regime. If, however, the electronic density is sufficiently high that the self-interaction is relevant, the dynamical regime is called Raman regime. Raman regimes involve space-charge plasma waves, whose resulting forces acting on the electronic population are comparable to the forces produced by the ponderomotive potential well. ${ }^{4-8}$

Space-charge effects in Raman regimes have been largely reported in the literature. Emphasis is mostly directed to issues related to instability and saturation levels of the electromagnetic mode, which are of central importance for FEL applications. ${ }^{9,10}$

More complete views of FELs also include careful analysis of particle dynamics, especially in Raman regimes where collective space-charge effects become more prominent. ${ }^{4}$

Relaxation in FELs occurs through dynamical mixing. If the charge is small, the ponderomotive well alone mainly drives the particle dynamics. In this case, the electronic distribution is attracted to the bottom of the ponderomotive well, revolving as a whole around itself in the particle phasespace.

When the charge increases, electric repulsion offers resistance against the ponderomotive effect. In this case, not only the relaxation is delayed with respect to Compton approximations, ${ }^{11}$ but also the way the system relaxes

\footnotetext{
a) peterpeter@uol.com.br

b) aendler@if.ufrgs.br

c)rizzato@if.ufrgs.br

d)serberto@if.uff.br
}

reveals new features associated with the presence of density waves. As far as particle dynamics is concerned, the setting is relatively similar to the wave-breaking process in the case of magnetically focused charged beams. ${ }^{12,13}$ The bunching action of the ponderomotive force in FELs indeed plays an equivalent role to the focusing action of the guiding magnetic field, and in both cases space-charge effects oppose the focusing drive.

The purpose of this work is to examine dynamics and relaxation through analysis of the particle phase-space, both in Compton and Raman regimes. We shall make use of an approach which proves effective in the analysis of magnetic focused beams. The approach is largely based on the compressibility factor, ${ }^{14}$ whose zeroes indicate the onset of mixing in phase-space. With help of the compressibility, we shall obtain an approximate, semi-analytical way, to calculate not only the time for the onset of mixing and subsequent relaxation but also the saturated amplitude of the radiation field. The approach also helps to identify the presence of space-charge effects in Raman regimes, as the compressibility develops characteristic oscillations in this case.

The paper is organized as follows: in Sec. II, we discuss the basic physical model; in Sec. III, we produce an analytical approximation for the resulting set of equations; in Sec. IV, we investigate the model and compare the analytical approximation with results from full simulations; and in Sec. $\mathrm{V}$, we draw our conclusions.

\section{THE PHYSICAL MODEL}

As mentioned earlier, particles interact with the electromagnetic mode and also directly among themselves. This latter self-interacting part of the dynamics relates to the system space-charge and the associated electric field.

Let us now briefly discuss the relevant fields and also the geometry of our system.

Laser and wiggler $(w)$ fields are described by the respective vector potentials $\mathbf{A}$ and $\mathbf{A}_{w}$, which we write in the form

$$
\frac{e \mathbf{A}}{m c^{2}}=a(z) \hat{\mathbf{e}} \exp i(k z-\omega t)+c . c .,
$$




$$
\frac{e \mathbf{A}_{w}}{m c^{2}}=a_{w} \hat{\mathbf{e}} \exp i\left(k_{w} z\right)+\text { c.c.. }
$$

The dimensionless laser amplitude, $a=a(z)$, is a slowly varying function of $z$, which we occasionally refer to as "time". The dimensionless wiggler amplitude $a_{w}$, on the other hand, is constant. Parameters $k$ and $\omega$ are, respectively, the wavenumber and frequency of the carrier laser mode in the tenuous medium, and $k_{w}$ is the wavenumber of the wiggler field which we assume to be of magnetostatic nature. The quantities $m$ and $e$ are, respectively, the electron mass and charge, and $c$ is the speed of light. We adopt circular polarization both for the laser and wiggler fields, with $\hat{\mathbf{e}}$ $=1 / \sqrt{2}(\hat{\mathbf{x}}+i \hat{\mathbf{y}})$ as the polarization versor. To simplify the entire discussion, we assume the laser to fill the cross sectional inner region of a wide pipe and therefore neglect transverse effects. This is why we use the one dimensional expression Eq. (1). ${ }^{6}$

As for the space-charge contribution, we consider a thin electron beam moving at the center of the pipe. The grounded walls of the pipe absorb exceeding lines of the electric field generated by the charged particles and allow periodic boundary conditions to be imposed. The more concrete physical picture is of a beam propagating along the $z$ axis of the coordinate system, with grounded plates located at $x= \pm L / 2$. Based on this simple sheet beam model, ${ }^{15}$ we solve Poisson equation and demand $2 \pi$ periodicity for the variable $\theta=k_{p} z-\omega t$, where $k_{p}=k+k_{w}$ is the wavenumber of the ponderomotive wave and $\theta$ is the particle phase in the ponderomotive potential. Since we are interested in longitudinal instabilities, we consider the beam to be uniform along the transverse coordinate $y$. Then, in the limit of large values of $L$, the space-charge electric field generated at $\theta$ by one particle of unitary charge located at $\theta^{\prime}$, can be represented by the periodic saw-tooth function

$$
E_{z}^{G}\left(\theta, \theta^{\prime}\right)=\operatorname{Sign}\left(\theta-\theta^{\prime}\right)\left[\pi-\operatorname{Abs}\left(\theta-\theta^{\prime}\right)\right] .
$$

This is actually the dimensionless Green's function for the electric field, graphically represented in Fig. 1, whose derivation is deferred to the Appendix. Hence, one can now express the full electric field at particle phase $\theta$ in the traditional Green's function form

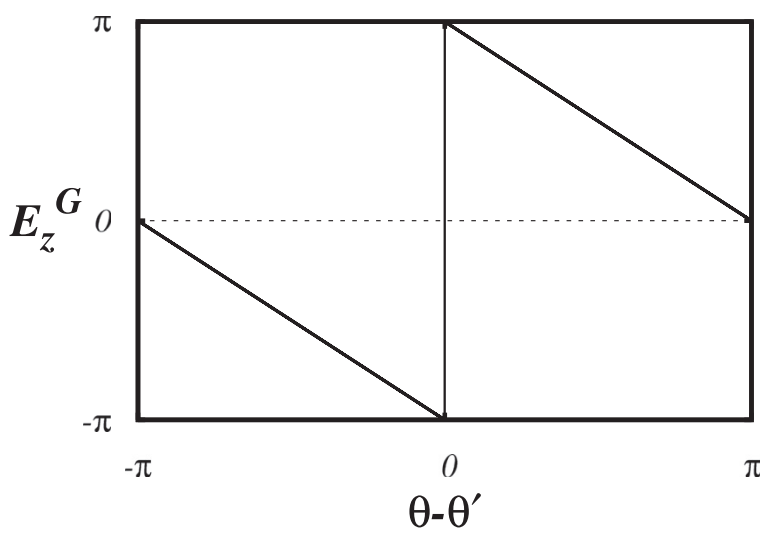

FIG. 1. Profile of the Green's function $E_{z}^{G}$.

$$
E_{z}(\theta)=\eta^{2} \frac{1}{N} \sum_{j} E_{z}^{G}\left(\theta, \theta_{j}\right) \equiv \eta^{2}\left\langle E_{z}^{G}\left(\theta, \theta_{j}\right)\right\rangle,
$$

where the summation runs over the total number of macroparticles, $N$, in the distribution: $j=1,2,3, \ldots, N$. Here, $\eta$ $=\sqrt{\omega_{p}^{2} / \omega^{2}}$ for fast beams where $k \approx k_{p}$, and $\omega_{p}^{2}$ $=4 \pi e^{2} n_{0} / m c$ is the plasma frequency with $n_{0}$ being the electron density averaged over the entire cross section of the pipe.

The expression given in Eq. (4) is the one actually used in the coming simulations. However, a more suitable form, convenient to handle analytically the space-charge electric field, can be obtained if one carefully manipulates the discontinuous behavior of the Green's functions as the source coordinates $\theta_{j}$ cross $\theta$. This alternative form reads

$$
E_{z}(\theta)=\pi \eta^{2}\left(\frac{N_{-}-N_{+}}{N}\right)+\eta^{2}\left(\left\langle\theta_{j}\right\rangle-\theta\right) .
$$

$N_{-(+)}$is the number of particles to the left (right) of coordinate $\theta, N=N_{-}+N_{+}$, and $\left\langle\theta_{j}\right\rangle$ is the center of mass of the distribution inside of the ponderomotive potential.

One sees that the space-charge electric field in Eq. (5) is essentially determined by two factors: the number of macroparticles to the right and left of the point where the field is evaluated, and an explicit dependence on the phase $\theta$. The former term involving the difference in the number of macroparticles is equivalent to terms appearing in charged sheet models. The latter term explicitly involving the phase difference assures that the proper periodic conditions on $\theta$ are satisfied, which is made possible by the truly multidimensional aspect of the problem. In the context of Fig. 1, for instance, the first term produces the discontinuous behaviour as one crosses a single sheet, while the second produces the adequate slopes to periodically match the solutions.

We shall return to Eq. (5) shortly, but for the present we write down the full set of equations which should be integrated. The first pair of equations, which describe the dynamics of each individual particle, is given by

$$
\begin{gathered}
\frac{d \theta_{j}}{d z}=\left(\frac{v_{z_{j}}}{v_{p}}-1\right), \\
\frac{d \gamma_{j}}{d z}=-\frac{a_{w}}{2 \gamma_{j}}\left(a e^{i \theta_{j}}+\text { c.c. }\right)+v_{z_{j}} v_{p} E_{z}\left(\theta_{j}\right),
\end{gathered}
$$

again for $j=1,2,3, \ldots, N$. We take into account only the space derivative, since we assume that the particle dynamics is on steady-state regime. The particle relativistic factor, $\gamma_{j}$, is given as

$$
\gamma_{j}=\frac{\sqrt{1+A_{t o t}^{2}}}{\sqrt{1-v_{z_{j}}^{2}}},
$$

with $\mathbf{A}_{t o t}=\mathbf{A}_{w}+\mathbf{A}$ denoting the total field. Variable $v_{z_{j}} / c \rightarrow v_{z_{j}}$ is the dimensionless electron velocity in the laboratory frame, $v_{p}=\omega / c k_{p} \approx k / k_{p}$ is the dimensionless phase velocity of the ponderomotive wave, and $k_{p} z \rightarrow z$. It should be noted that the particle phase $\theta_{j}$ remains constant if the 
particle velocity, $v_{z_{j}}$, is the same as the ponderomotive phase velocity, $v_{p}$.

As in Refs. 6,16, and 17, the stimulated radiation amplitude, $a$, is governed by the following slowly varying envelope approximation for the wave equation, viz.,

$$
\frac{d a}{d z}=\eta^{2} v_{p} a_{w}\left\langle\frac{e^{-i \theta_{j}}}{2 \gamma_{j}}\right\rangle-i \eta^{2} v_{p}\left\langle\frac{1}{2 \gamma_{j}}\right\rangle a,
$$

with the brackets again indicating an average over the electron distribution.

This set of nonlinear equations, Eqs. (3)-(9), describes completely the dynamics of a FEL with a constant wiggler parameter, $a_{w}$, taking into account the presence of spacecharge effects.

\section{OVERVIEW AND SIMPLIFIED WAVE-PARTICLE DYNAMICS}

In this section, we shall develop a simple semianalytical model in order to provide a reasonable footing for the investigation that we wish to conduct.

Let us consider that the stimulated radiation is amplified from small values, and that at $z=0$, particles are distributed uniformly over the phase $\theta$, traveling along the $z$-axis with a velocity $v_{0}$. We take $v_{0}$ to be in the vicinity of, but not necessarily equal to, the ponderomotive wave phase velocity $v_{p}$. There is, therefore, room for a detuning between the radiation and the particle beam. The detuning shall always be chosen to optimize the FEL interaction, maximizing the growth rate of the stimulated radiation.

During the interaction, the particles become subjected to the combined action of the ponderomotive and space-charge forces. The relative magnitude between these two forces plays a crucial role in the operation regime of the FEL, which can be the Raman regime, if the space-charge force intensity is comparable to the ponderomotive force, or the Compton regime, if the space-charge force is smaller than the ponderomotive one. In any case, as time evolves, the particle beam undergoes phase-mixing in the phase-space, and the whole process reaches saturation afterwards. ${ }^{6}$

As known, the onset of mixing process occurs before the saturation of the amplified radiation is established. Hence, we can approximately describe the stimulated mode as initially evolving according to its linear stage of amplification. This approximation does not hold at all times, but should provide a relatively accurate description of the radiation field up to the initial stages of the mixing process.

In order to describe the particle dynamics we go one step further, refining the linear approximation and keeping nonlinearities up to dominant order. This latter step in our approach creates a slight but necessary contrast with the case of static magnetic focusing discussed earlier. While magnetically focused beams move relatively near their equilibrium engendered by the balance of the static focusing and space-charge forces, ${ }^{18,19}$ here, particles are never in equilibrium with the growing laser field. Therefore, inclusion of nonlinear corrections offers better agreement with simulations.

\section{A. Nonlinear particle dynamics}

To build our semi-analytic model, we start with the relatively less explored particle dynamics as described by Eqs. (6) and (7). It should be noted that the relativistic factor, $\gamma_{j}$, appears in Eq. (6) through the particle velocity $v_{z_{j}}$. The stimulated radiation grows from small initial conditions and one can use Eq. (8) to expand the velocity $v_{z_{j}}$ in terms of the small variations of the relativistic factor $\gamma_{j}$ and of the radiation amplitude itself. For relativistic beams, $\gamma_{j}$ becomes a sensitive function of $v_{z_{j}}$ and the corresponding term dominates the expansion. We point out that the expansion, when inserted in right-hand-side of Eq. (6), generates a small zero order term of the form $\left(v_{0}-v_{p}\right) / v_{0}$, which we define as the detuning $\nu$. If the detuning is absorbed into a redefined $\theta \rightarrow \theta+\nu z$, and the radiation field is rescaled as $a \rightarrow a e^{-i \nu z}$, the resulting system takes the form of a resonant system with $v_{0}=v_{p}$, but with an extra term $i \nu a$ added to the right-hand-side of Eq. (6). We shall adopt these rescalings to describe the simplified system.

In order to have a simpler form, now for Eq. (7), we approximate $\gamma_{j} \approx \gamma_{r}$ and $v_{z_{j}} \approx v_{p}$ on the right-hand-side, keeping the remaining form of the ponderomotive term intact. Here, $\gamma_{r}$ is the resonant normalized energy of the electrons.

The electric field, $E_{z}\left(\theta_{j}\right)$, present in the second term on the right-hand side of Eq. (7), is obtained with the help of the expression given by Eq. (5). Let us, therefore, carry out the respective calculation in the following approximate way. Up to the beginning of the mixing process where particles overtake each other, the particle population also remains approximately confined within the region $-\pi<\theta_{j} \leq+\pi$. The numbers $N_{-} / N$ and $N_{+} / N$ are thus approximate constants. They can be cast in terms of the uniform initial conditions as $N_{\mp} / N=\left|\theta_{0} \pm \pi\right| / 2 \pi$, if one uses simple proportionality arguments. The phase $\theta_{0}$ denotes the initial position of the particle which we are keeping track of, and we are assuming that the particle distribution remains acceptably uniform up to mixing. Therefore, here we set $\left\langle\theta_{j}\right\rangle \rightarrow 0$ in Eq. (5) for the electric field.

If we now introduce $D_{\gamma} \equiv\left(\partial v_{z_{j}} / \partial \gamma_{j}^{2}\right)_{\text {injection }}$, then the simplified equation describing the particle dynamics takes the form

$$
\begin{aligned}
\frac{d^{2} \theta_{j}}{d z^{2}}= & -\frac{D_{\gamma}}{v_{p}}\left[a_{w} \tilde{a}(z) e^{i \theta_{j}(z)}+c . c .\right] \\
& +2 v_{p} \eta^{2} D_{\gamma} \gamma_{r}\left(\theta_{0}-\theta_{j}(z)\right),
\end{aligned}
$$

which gives the particle phase, $\theta_{j}$, with an explicit dependence on the initial particle phase, $\theta_{0}$. Here, $\tilde{a}$ represents the linear approximation of the stimulated radiation amplitude, rescaled as discussed earlier. Once again we note that subscript $j$ is dropped from $\theta_{0}$ to simplify the notation, but that each $\theta_{j}$ evolves from its initial $\theta_{0}$ of the uniform distribution.

Deriving Eq. (10) with respect to $\theta_{0}$, and defining $\partial \theta_{j} / \partial \theta_{0} \equiv C$ as the compressibility, ${ }^{14}$ we obtain an evolution equation with which one can identify the beginning of the phase-mixing process in the FEL, namely,

$$
\begin{aligned}
\frac{d^{2} C}{d z^{2}}= & -\frac{D_{\gamma}}{v_{p}}\left[i C(z) a_{w} \tilde{a}(z) e^{i \theta_{j}(z)}+c . c .\right] \\
& +2 v_{p} \eta^{2} D_{\gamma} \gamma_{r}(1-C(z)) .
\end{aligned}
$$


As we can see, the compressibility $C$ depends on the initial phase $\theta_{0}$ and on $z$. If $C\left(z, \theta_{0}\right) \rightarrow 0$, particles located in the vicinity of $\theta_{0}$ when $z=0$, overtake each other at time $z$ and at the phase coordinate $\theta_{j}=\theta_{j}\left(z, \theta_{0}\right)$. Particles coming from slightly different $\theta_{0}$ s thus converge to the same $\theta$, the signature of mixing process in phase-space, and soon afterwards the system relaxes. We should point out that the presence of zeros of the compressibility is a local feature that makes explicit mention to the particle phases. This is the reason why, in our particle dynamics approximation, we need the local expressions given by Eqs. (10) and (11).

\section{B. Linear wave dynamics}

In order to solve the local dynamics described by Eqs. (10) and (11) in closed form, we should determine the evolution equation for the linearized radiation amplitude $\tilde{a}=\tilde{a}(z)$. Let us assume that the particle phase, $\theta_{j}$, and normalized particle energy, $\gamma_{j}$, can be written as $\theta_{j}=\theta_{0}+\delta \theta_{j}$ and $\gamma_{j}=\gamma_{r}+\delta \gamma_{j}$, where $\delta \theta_{j}$ and $\delta \gamma_{j}$ are first order perturbations, respectively. Hence, introducing these into Eqs. (6)-(9) and making use of the collective variable description developed in Ref. 6, we obtain the following linearized wave equation, namely,

$$
\frac{d \tilde{a}}{d z}=-i \frac{v_{p} \eta^{2}}{2 \gamma_{r}} a_{w} X-\frac{v_{p} \eta^{2}}{2 \gamma_{r}^{2}} a_{w} Y-i \frac{v_{p} \eta^{2}}{2 \gamma_{r}} \tilde{a}+i \nu \tilde{a}
$$

where $X=\left\langle\delta \theta_{j} e^{-i \theta_{0}}\right\rangle$ and $Y=\left\langle\delta \gamma_{j} e^{-i \theta_{0}}\right\rangle$ are given by

$$
\begin{gathered}
\frac{d X}{d z}=\frac{2}{v_{p}} D_{\gamma} \gamma_{r} Y-i \frac{a_{w}}{v_{p}} D_{A} \tilde{a}, \\
\frac{d Y}{d z}=-v_{p}^{2} \eta^{2} X-\frac{1}{2 \gamma_{r}} a_{w} \tilde{a} .
\end{gathered}
$$

Here, we introduce $D_{A}=\left(\partial v_{z_{j}} / \partial A_{\text {tot }}^{2}\right)_{\text {injection }}$ and observe the presence of the detuning in the equation for the rescaled radiation field, Eq. (12).

For given values of $a_{w}$ and $v_{0}$, the linear set represented above is unstable in a limited region of the $\nu$ versus $\eta$ parameter space, as shown in Fig. 2. Not clear in the figure is the fact that the unstable region extends to increasingly negative values of $\nu$ as $\eta \rightarrow 0$. The figure also displays the curve of maximum growth rate (dots) with the maximization procedure performed over $\nu$ at each level $\eta$. The maximum growth rate curve is the one selecting the dominant modes of the interaction.

To obtain the figure we set $a_{w}=0.4, v_{0}=0.99$ and, unless stated otherwise, use these values for $a_{w}$ and $v_{0}$ throughout the paper, along with a seeding, small initial condition for $a: \tilde{a}(z=0)=-i a_{w} \times 10^{-5}$.

The linear equations (12)-(14) derived above can now be combined with Eqs. (10) and (11) to evaluate the compressibility, $C$. The compressibility will be used to calculate the time required for the onset of mixing, which provides a way to make estimates on the saturated amplitude of the radiation. The compressibility will also allow to investigate the presence of space-charge plasma waves in the waveparticle dynamics.

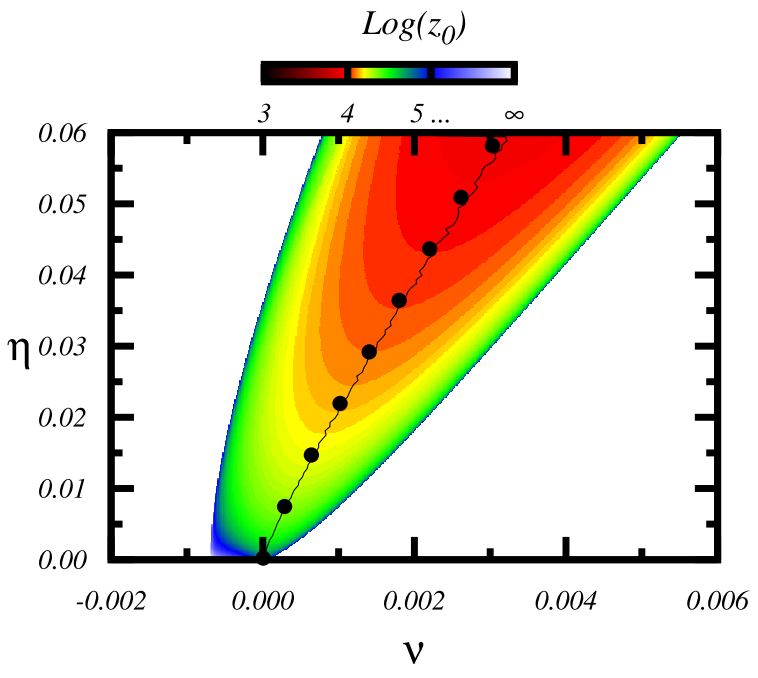

FIG. 2. Colour graded unstable region in the $\nu, \eta$ plane. White represents stability and the different colours are associated with the magnitude of $z_{0}$ through the labelling. The large dots represent the parameters for the maximum growth rate curve, and the full line represents the parameters for minimum time for the onset of mixing.

\section{ANALYSIS OF THE SIMPLIFIED MODEL AND SIMULATIONS}

\section{A. Onset of mixing}

To investigate the onset of mixing in the general case of arbitrary parameters $\eta$ and $\nu$, we follow the behaviour of the compressibility as a function of the time $z$ and take note of the earliest instant where, for some $\theta$, it goes to zero-we call it $z_{0}$. If one now minimizes this time over $\nu$, the solid, slightly wavy line of Fig. 2 is produced. One sees that, for all practical purposes, both mixing and maximum growth curves are coincident: the dominant fastest growing modes are also the ones commanding the mixing process.

A figure is then constructed when we plot $z_{0}$ as a function of $\eta$, with the proviso that the detuning $\nu$ is always chosen such that one moves along the curve of maximum growth rate of the linear instability. As mentioned earlier, this latter condition selects the dominant mode of the stimulated radiation. All the subsequent analysis performed in the paper will be restricted to the maximum growth rate curve.

Our results are summarized in Fig. 3, where simulations (performed with 1000-5000 particles) are compared with the corresponding results of our semi-analytical model. We see that the agreement is reasonable and that the time for mixing monotonically decreases as the charge $\eta$ increases.

Mixing is subsequently accompanied by relaxation. Since we have a way to calculate the time related to the former, we now attempt to estimate the amplitude relative to the latter. In terms of the model, the relaxed amplitude of the laser is simply estimated as the linear amplitude obtained from the simplified model, evaluated at the mixing onset time $z_{0}$; $a_{\text {mod }}^{\text {sat }} \equiv\left|\tilde{a}\left(z=z_{0}\right)\right|$. The corresponding relaxed amplitude arising from the simulations, $a_{\text {sim }}^{\text {sat }}$, is calculated as we perform a time average of the amplitude over a relatively large time stretch following the onset of mixing. Our choice here is to perform the average from $z_{0}$ to $3 z_{0}$. Results are plotted in Fig. 4 and again indicate good agreement between the two 


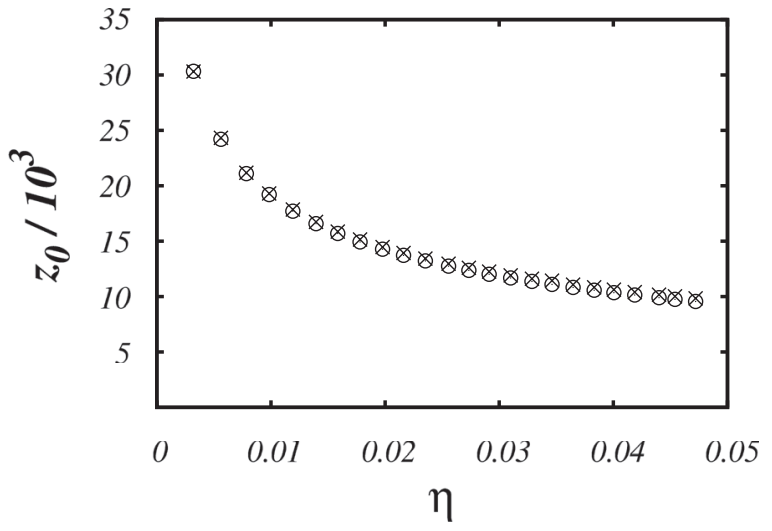

FIG. 3. Behavior of $z_{0}$ versus $\eta$ both from the semi-analytical model (crosses) and simulation (circles).

approaches. A more judicious look at Fig. 4 actually shows that the two curves begin to move apart at larger values of the charge and field. The discrepancy is related with the approximations leading to Eqs. (10) and (11), and with the assumption of a linear dynamics for the laser field: any small error in the onset time leads to relatively larger errors in the estimates of the exponentially growing stimulated radiation. In any case, we believe that the estimates made here still keep a reasonable balance between accuracy and simplicity.

\section{B. Space-charge effects}

We finally discuss the role of space-charge in our model. To do that, once again we find the compressibility a helpful tool to look at the subject.

In Fig. 5, we plot $C$ [Eq. (11)] versus $z$ for two instances: in panel (a), we consider $\eta=0.001$ and in panels (b) and (c), $\eta=0.05$. While in panel (a), $C$ simply describes a descending trajectory to zero following a small growth; in panel (b), one observes a number of oscillations prior the ultimate downward path of $C$. Panel (c) magnifies the plot seen in (b) and allows to observe more clearly the oscillatory behaviour of the compressibility. A black dot is placed at the same coordinate in panels (b) and (c) - a peak of oscillation-to help, to correlate both panels, and keep track of $C$. If for the larger value of $\eta$ one turns off the space-charge term in Eq. (7) (and the respective detuning), a figure similar to that of

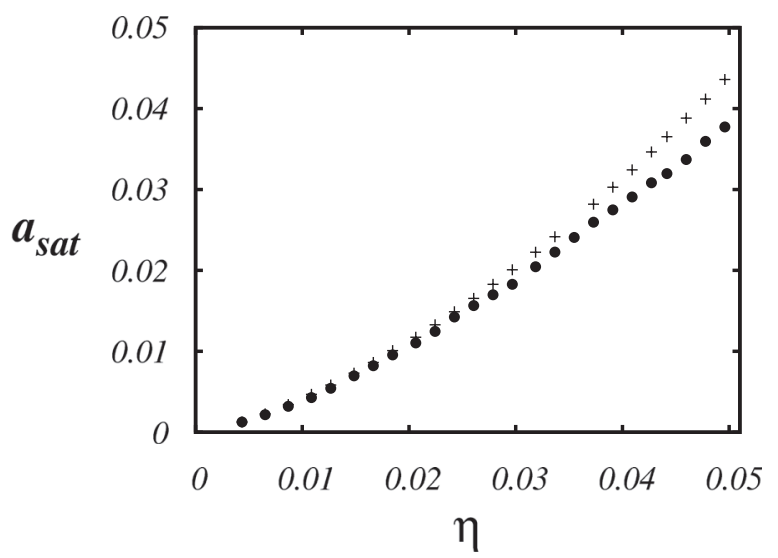

FIG. 4. Saturated amplitudes of the stimulated laser field, again obtained from the semi-analytical model (crosses) and simulations (filled circles).

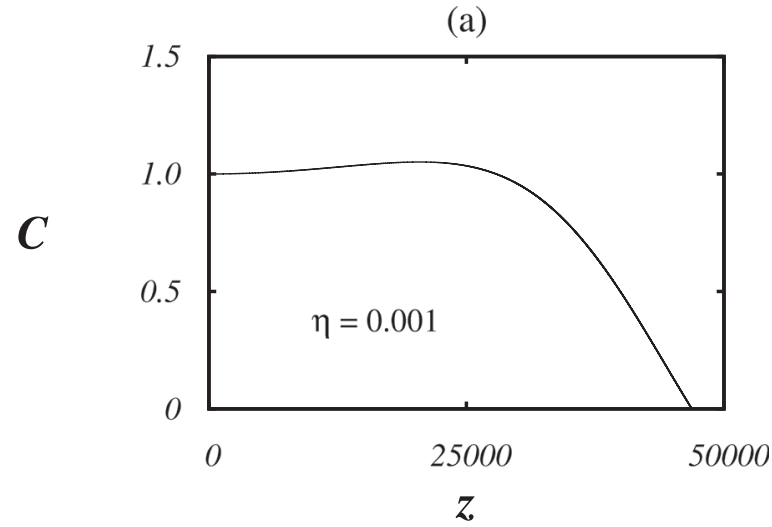

(b)
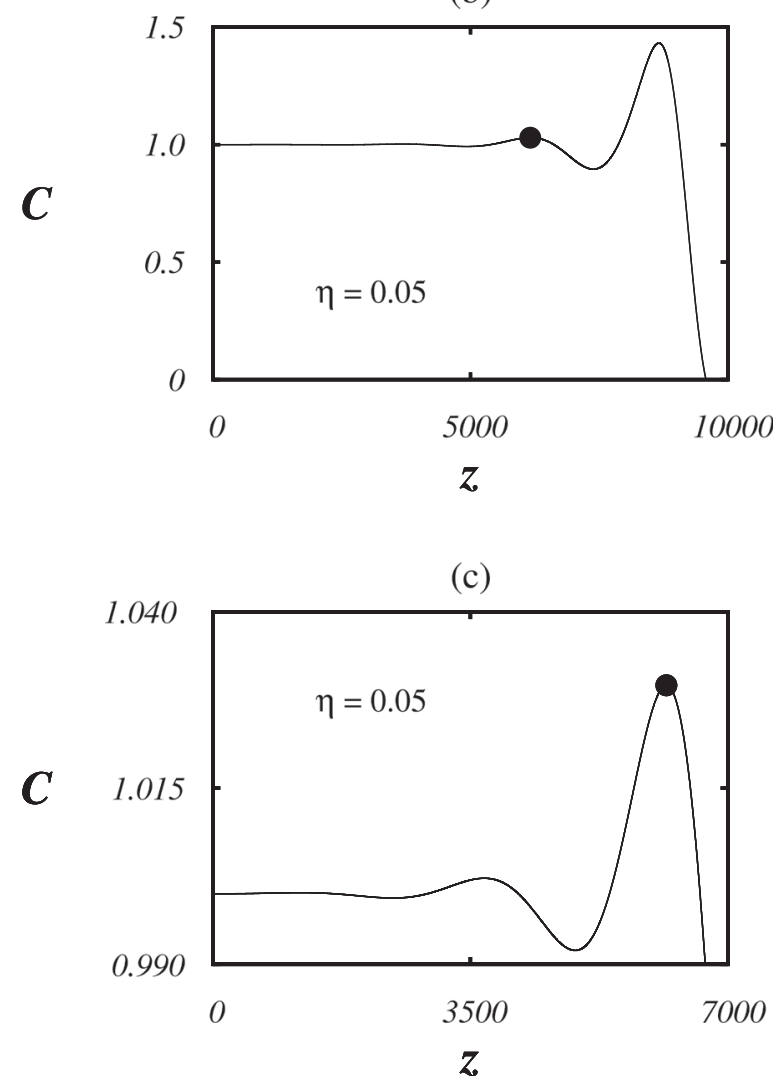

FIG. 5. Behavior of $C$ versus $z$ for a small $\eta, \eta=0.001$ in panel (a), and a large $\eta, \eta=0.05$ in panels (b) and (c). Panel (c) magnifies the view of panel (b) to clearly display the corresponding oscillatory cycles, absent when the charge is small.

panel (a) is obtained, which suggests that the oscillations involve charge-density waves. As the charge increases, there is one particular critical value for $\eta, \eta_{c}$, where one full oscillatory cycle is added to the initial pattern represented in panel (a). We consider one cycle to be fully added, when the next cycle is about to appear in the $C$ versus $z$ plot.

In the case $a_{w}=0.4$, we are investigating $\eta_{c}\left(a_{w}=0.4\right) \approx 0.0074$. This implies in $\left|a_{\text {mod }}^{\text {sat }}\right| \sim 0.0025$, which yields approximately a unitary ratio of the ponderomotive to the space-charge terms in Eq. (7). For larger values of $\eta$ the ratio decreases, and for smaller values, it increases. At the transition both terms are thus comparable, and from then on space-charge terms can no longer be discarded. 


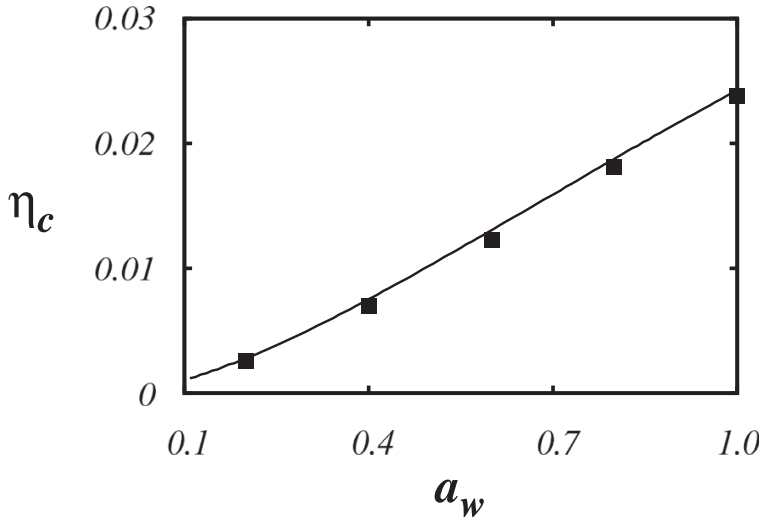

FIG. 6. Behavior of $\eta_{c}$ versus $a_{w}$. Semi-analytical model in solid line, and simulations represented by filled squares. The curve can be seen as the interface between the Compton (below) and Raman (above) regions.

In Fig. 6, we extend the analysis of $\eta_{c}$ to other values of $a_{w}$. A number of points obtained from simulations are superposed to the solid line obtained with the semi-analytical model, from which a general concurrence of results is observed. The case $a_{w}=1$ has been briefly discussed in Ref. 6. For $v_{p}=0.99$, the chosen value of $\eta$ to illustrate space-charge effects reads 0.04 , which is indeed already in the space-charge region obtained with the present analysis $\eta_{c}\left(a_{w}=1.0\right) \sim 0.025$.

The final topic we wish to discuss is on the structure of the particle phase-space. Returning to $a_{w}=0.4$ we compare

(a)

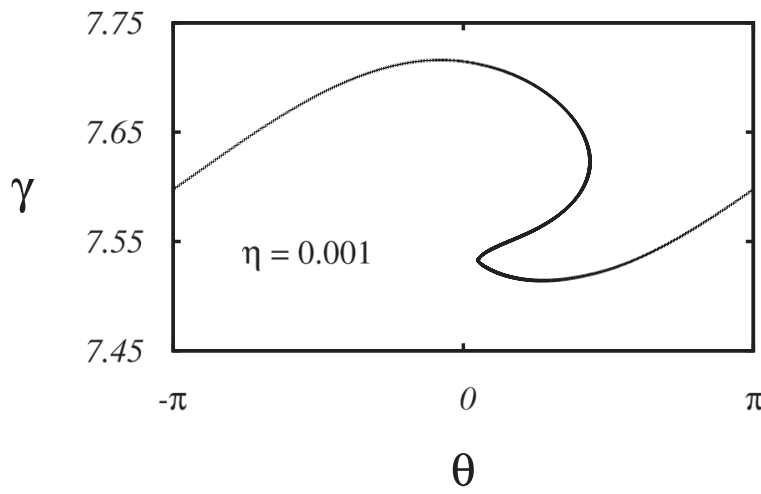

(b)

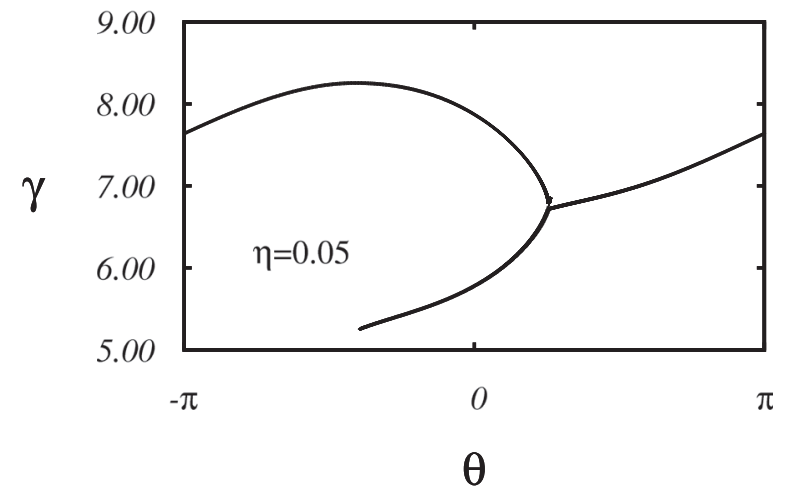

FIG. 7. Snapshots of the particle phase-space as obtained from numerical simulations. In (a), $\eta=0.001<\eta_{c}$; and in (b), $\eta=0.05>\eta_{c}$. two cases: $\eta=0.001<\eta_{c}$ illustrating the Compton regime, and $\eta=0.05>\eta_{c}$ representing the Raman regime. The Compton case is displayed in panel (a) of Fig. 7, and the Raman case in panel (b) of the same figure.

Both snapshots are taken immediately after the onset of mixing. In the Compton case, relaxation proceeds as the particle distribution mostly revolves as a whole around itself in phase-space. ${ }^{4}$

In the Raman case, however, relaxation is better described in terms of jets emanating from the core of the particle distribution, as in core-halo systems. ${ }^{20}$ Jets are formed by charge density piling up, which creates regions of singular acceleration. Jets are not actually absent in the Compton regime, but due to low amount of charge, they are overshadowed by the ponderomotive acceleration.

Even though the ponderomotive well is not stationary, the dynamics at low and high values of $\eta$, respectively, bear some resemblance to the dynamics of fast and slow wavebreaking in magnetostatically confined beams. We recall that fast wave-breaking occurs when magnetic focusing is strong and waves break in less than an oscillatory cycle, with slow wave-breaking occurring only after a series of oscillatory cycles. ${ }^{13,21}$

\section{CONCLUSIONS}

In the present paper, we have investigated dynamics and relaxation in free-electron lasers, with the help of tools which proved useful in similar systems. The analysis has been mainly performed with the help of the compressibility, whose zeroes indicate the onset of mixing in the particle phase-space. The compressibility has recently been used to investigate properties of magnetostatically confined relativistic beams,${ }^{14}$ and in the present situation it not only allows to analytically determine the onset of mixing, but also to estimate the relaxed amplitude of the radiation field.

All the quantities analytically obtained with the compressibility compare well with the wave-particle simulations performed in the work.

The compressibility also offers a way to look at spacecharge effects. As one examines plots of $C$ versus $z$ for various values of $\eta$, one finds out that when $\eta$ is small, $C$ drops to zero from its initial value $C=1$ without executing full oscillatory cycles. This is the pattern also seen, even for larger values of $\eta$, if space-charge terms are turned off in Eq. (7). On the other hand, when $\eta$ is large, $C$ drops to zero only after a number of full oscillatory cycles. For given values of $a_{w}$ and $v_{p}$, there is thus a critical value of $\eta$ where one full cycle is just added to the initial low charge pattern. As illustrated in the text, at this point, the magnitude of the space-charge and ponderomotive terms in Eq. (7) become comparable. Larger (smaller) values of $\eta$ increase (decrease) the ratio of the space-charge term to the ponderomotive term.

Relevant effects due to thermal effects and the transverse structure of the stimulated radiation are neglected in our model and should be investigated in the near future. In particular, a recent work on thermal effects suggests that even very small temperatures can be used to control the mixing time. ${ }^{14}$ 


\section{ACKNOWLEDGMENTS}

This work was supported by $\mathrm{CNPq}$ and FAPERGS, Brazil, and by the Air Force Office of Scientific Research (AFOSR), USA, under the Grant No. FA9550-12-1-0438. The authors thank useful discussions with Renato Pakter.

\section{APPENDIX: GREEN'S FUNCTION FOR THE SPACE CHARGE FIELD}

Recalling our set of dimensionless variables and the definition of $\theta$, we start by writing the dimensionless Green's equation for the space-charge potential in the convenient form

$$
\nabla^{2} G(\theta, x)=-2 \pi\left(\frac{L}{2}\right) \delta\left(\theta-\theta_{0}\right) \delta(x),
$$

with $\nabla^{2} \equiv \frac{\partial^{2}}{\partial \theta^{2}}+\left(\frac{1}{k_{p}}\right)^{2} \frac{\partial^{2}}{\partial x^{2}}$. Once $G$ is found, the longitudinal component of the full electric field based on Gauss law can be written as in Eq. (5). The multiplicative constant $\eta$ measures the magnitude of charge effects, and $E_{z}^{G}=-\partial G / \partial \theta$.

Considering grounded plates at $x= \pm L / 2$, we expand the solution of Eq. (A1) in an orthogonal basis as

$$
G(\theta)=\sum_{n=1}^{\infty} G_{n}(\theta) \cos \left(\frac{(2 n+1) \pi x}{L}\right),
$$

alongside with the expansion

$$
\delta(x)=\frac{2}{L} \sum_{n=0}^{\infty} \cos \left(\frac{(2 n+1) \pi x}{L}\right) .
$$

From Eqs. (A2) and (A3), it follows:

$$
\left[\frac{\partial^{2}}{\partial \theta^{2}}-\left(\frac{(2 n+1) \pi}{k_{p} L}\right)^{2}\right] G_{n}(\theta)=-2 \pi \delta\left(\theta-\theta_{0}\right) .
$$

Solutions of Eq. (A4) for $\theta \neq \theta_{0}$ have exponential form and must be matched across $\theta_{0}$. In addition, we demand periodicity with $G_{n}(\theta=-\pi)=G_{n}(\theta=\pi)$ and $\partial G_{n}(\theta=-\pi) / \partial \theta$ $=\partial G_{n}(\theta=\pi) / \partial \theta$.

Algebraic work is required at this point, and solutions can be obtained. If we take only $n=0$ in the summation, and consider large values of $L$, the result for the corresponding electric field $E_{z}^{G}$ is represented by Eq. (4). Inclusion of higher values of $n$ only causes slight and smooth curvatures on the otherwise straight lines of the $n=0$ approximation.

${ }^{1}$ T. C. Marshall, Free-Electron Lasers (Macmillan Publishing Company, New York, 1985).

${ }^{2}$ C. Brau, Free-Electron Lasers (Academic Press, London, 1990).

${ }^{3}$ H. P. Freund and T. M. Antonsen, Principles of Free-Electron Lasers (Chapman \& Hall, London, 1996).

${ }^{4}$ P. Sprangle, C.-M. Tange, and W. M. Manheimer, Phys. Rev. A 21, 302 (1980).

${ }_{5}^{5}$ J. B. Murphy, C. Pellegrini, and R. Bonifacio, Opt. Commun. 53, 197 (1985).

${ }^{6}$ R. Bonifacio, F. Casagrande, G. Cerchoni, L. de Salvo Souza, P. Pierini, and N. Piovella, Riv. Nuovo Cimento 13, 1 (1990).

${ }^{7}$ Y. Seo and E. H. Choi, IEEE Trans. Plasma Sci. 25, 360 (1997).

${ }^{8}$ N. M. Kroll and W. A. McMullin, Phys. Rev. A 17, 300 (1978).

${ }^{9}$ G. Marcus, E. Hemsing, and J. Rosenzweig, Phys. Rev. ST Accel. Beams 14, 080702 (2011).

${ }^{10}$ L. F. Monteiro, A. Serbeto, K. H. Tsui, J. T. Mendonça, and R. M. O. Galvão, Phys. Plasmas 20, 073101 (2013).

${ }^{11}$ M. Wang, X. Xiao, and Z. Liang, J. Phys. D: Appl. Phys. 39, 3332 (2006).

${ }^{12}$ F. B. Rizzato, R. Pakter, and Y. Levin, Phys. Plasmas 14, 110701 (2007).

${ }^{13}$ E. G. Souza, A. Endler, R. Pakter, F. B. Rizzato, and R. P. Nunes, Appl. Phys. Lett. 96, 141503 (2010).

${ }^{14}$ E. G. Souza, A. Endler, F. B. Rizzato, and R. Pakter, Phys. Rev. Lett. 109, 075003 (2012).

${ }^{15}$ F. B. Rizzato, J. Plasma Phys. 44, 33 (1990).

${ }^{16}$ F. B. Rizzato, Phys. Rev. A 41, 1629 (1990); G. I. de Oliveira, F. B. Rizzato, and A. C. Chian, Phys. Rev. E 52, 2025 (1995).

${ }^{17} \mathrm{~J}$. T. Mendonça, Theory of Photon Acceleration (IOP Publishing, Bristol, 2001).

${ }^{18}$ C. Chen and R. C. Davidson, Phys. Rev. E 49, 5679 (1994).

${ }^{19}$ R. Pakter and F. B. Rizzato, Phys. Rev. Lett. 87, 044801 (2001); Phys. Rev. E 65, 056503 (2002).

${ }^{20}$ A. Antoniazzi, D. Fanelli, J. Barré, P.-H. Chavanis, T. Dauxois, and S. Ruffo, Phys. Rev. E 75, 011112 (2007).

${ }^{21}$ O. A. Anderson, Part. Accel. 21, 197 (1987). 